\title{
THREE-DIMENSIONAL MANIFOLDS AND THEIR HEEGAARD DIAGRAMS*
}

\author{
BY \\ JAMES SINUER \\ INTRODUCTION
}

One of the outstanding problems in topology today is the classification of $n$-dimensional manifolds, $n \geqq 3$. Poincaré, the founder of modern analysis situs, devoted several papers to it and allied problems. $\dagger$ Heegaard $\ddagger$, in a paper concerned primarily with another aspect of the subject, found it convenient to construct a pseudo-normal form for a 3-dimensional manifold, a form which we now call the Heegaard diagram. Dehn $\S$ and Veblen $\|$ gave modifications of his construction.

The Heegaard diagram of a 3-dimensional manifold consists of a closed 2-dimensional manifold upon which are drawn a certain number of nonintersecting simple closed curves. Any diagram is an adequate representation of a 3-dimensional manifold in the sense that it completely determines such a manifold, but, unfortunately, a 3-dimensional manifold gives rise to an infinity of diagrams. The problem of classifying manifolds is thus transferred to the problem of classifying diagrams.

Heegaard, in the paper cited above, studied (although not completely) the modifications that can be made on the curves and surface of a diagram which transformed it into another diagram but yet did not change the manifold which it represented. In this paper we extend Heegaard's results and study more completely the relationships between manifolds and their diagrams.

We begin then (Part I) by introducing the notions of a canonical region, canonical surface and canonical curve of a manifold. The Heegaard diagram is then constructed from a canonical surface and curves. Then, before proceeding to a discussion of manifolds and their diagrams, we show (Part II) how to read off the usual invariants of a manifold from any one of its representative diagrams.

* Presented to the Society, October 29, 1932; received by the editors June 10, 1932.

$\dagger$ Poincaré, H. For references see his paper in the Rendiconti del Circolo Matematico di Palermo, vol. 18 (1904).

$\ddagger$ Heegaard, P. A translation into French is given in the Bulletin de la Societe Mathematique de France, vol. 44 (1916).

$\S$ Dehn, M., Mathematische Annalen, vol. 69 (1910).

|| Veblen, O., The Cambridge Colloquium, Analysis Situs, 2d edition, p. 155. 
We then define (Part III) a set of moves which operate on the curves and surface of a diagram and transform it into another. Two diagrams are called equivalent if one can be obtained from the other by a finite number of these moves. We then prove by a sequence of theorems (Part IV) in which we make use of a specially constructed canonical surface and diagram that any two representative diagrams of a manifold are equivalent. Several other theorems (Part V) lead up to the general theorem of equivalence to the effect that equivalent manifolds (equivalent in the sense of semi-linear analysis situs) arise from and give rise to equivalent Heegaard diagrams (equivalent in our sense) and vice versa.

I wish to thank Professor J. W. Alexander for his many suggestions in the preparation of this paper.

\section{Preliminary definitions}

1. We will assume that the reader is familiar with the simplex, complex, manifold, incidence matrix, etc., as used in combinatorial analysis situs as, for example, in the book by Veblen, loc. cit. Throughout this paper, simplexes, cells, etc., will be at most 3-dimensional.

2. We derive here several elementary properties of the simplex which we will need later. Let $\bar{\sigma}_{3}=A^{0} A^{1} A^{2} A^{3}$ be a closed 3-simplex, $\sigma_{k}, \sigma_{2-k}(k=0$ or 1$)$ two opposite faces of $\sigma_{3}$ (e.g., $\sigma_{k}=A^{0}, \sigma_{2-k}=A^{1} A^{2} A^{3}$ ); let $\bar{\sigma}_{3}^{\prime}$ be the derived complex (i.e., a regular subdivision) of $\bar{\sigma}_{3}$. Every 3-simplex of $\sigma_{3}^{\prime}$ contains a vertex of $\sigma_{k}$ or of $\sigma_{2-k}$, but not of both. It follows at once that all the 3-simplexes of $\bar{\sigma}_{3}^{\prime}$ fall into two groups, $R_{1}$ and $R_{2}$, where $R_{1}$ contains all the simplexes incident with $\sigma_{k}$ and $R_{2}$ contains all those incident with $\sigma_{2-k}$. Moreover, $R_{1}$ and $R_{2}$ have as boundaries $B_{1}+B$ and $B_{2}+B$, respectively, where $B_{1}$ and $B_{2}$ consist of 2 -simplexes of $\bar{\sigma}_{3}^{\prime}$ on the boundary of $\bar{\sigma}_{3}$, and the common portion $B$ is a 2 -cell whose boundary is a circuit on the boundary of $\bar{\sigma}_{3}$ which "separates" $\sigma_{k}$ and $\sigma_{2-k}$.

3. The canonical region, cell and curve. Given a 3-cell with its, boundary sphere $S_{2}$, we may decompose $S_{2}$ into a sum $E_{2}^{\prime}+E_{2}^{\prime \prime}+\bar{B}$ where $E_{2}^{\prime}$ and $E_{2}^{\prime \prime}$ are two cells such that $\bar{E}_{2}^{\prime}$ and $\bar{E}_{2}^{\prime \prime}$ do not meet and $B$ is a spherical band.* We can think of this system as a handle with the $E$ 's as its bases.

We will now take a euclidean 3-sphere and attach $p$ handles to it thus obtaining a 3-dimensional region $R$ which we call canonical. Explicitly, we have $R=E_{3}^{0}+\sum_{i=1}^{p}\left(E_{3}^{i}+E_{2}^{\prime i}+E_{2}^{\prime \prime}{ }^{i}\right)$ where $E_{3}^{0}$ is the spherical region and the elements in the sum represent the handles and their bases. The (point set)boundary of $R$ will be designated by $L . \dagger$ Incidentally, $L$ is not to be construed

* The bar over a symbol for a set denotes the closure of the set.

$\dagger$ We shall hereafter omit the words point set, understanding that whenever we speak of the boundary of a canonical region, we mean the (point set)-boundary. 
to lie in euclidean 3-space, for it may very well happen that $L$ is non-orientable.

We recognize in $R$ the following property: if we sever each handle by a cross cut in the form of a 2-cell $E_{2}{ }^{i}$, what is left is a 3-cell. In other words we can think of a canonical region as a region $R$ within which there are $p 2$-cells $E_{2}^{i}$, with boundaries $e^{i}$ on the boundary $L$ of $R$ (no two $\bar{E}$ 's intersecting) such that $R-\sum E_{2}{ }^{i}$ is a 3 -cell. The cells $E_{2}{ }^{i}$ will be called canonical cells and their boundaries canonical curves.

4. The canonical surface and the Heegaard diagram. A surface $L$ is said to be a canonical surface of a manifold $M$ if it satisfies these conditions:

(a) $L$ is a subcomplex of $M$ and is a closed, connected 2-dimensional manifold;

(b) $I I$ can be decomposed into $R_{1}+L+R_{2}$, where $R_{1}$ and $R_{2}$ are canonical regions with the common boundary $L$.

We note four properties of the canonical surface and regions which follow directly from their definitions:

A. If $M^{\prime}$ is a subdivision of $M$, and $L^{\prime}, R_{1}^{\prime}$ and $R_{2}^{\prime}$ the induced subdivisions of $L, R_{1}$ and $R_{2}$, then $L^{\prime}$ is a canonical surface of $M^{\prime}$ dividing it into the canonical regions $R_{1}^{\prime}$ and $R_{2}^{\prime}$. We will also call $L^{\prime}$ a canonical surface of $M$.

B. The number of 2-cells that must be removed from $R_{1}$ to reduce it to a 3 -cell is the same as the number that must be removed from $R_{2}$, and each is precisely the maximum number of non-intersecting circuits that can be drawn on $L$ without disconnecting it (Heegaard).

C. $R_{1}$ and $R_{2}$ are homeomorphic, since they have the same boundary.

D. $L$ and $M$ are both orientable or non-orientable (Heegaard).

5. A problem as yet unsolved is the determination of the minimum genus (or connectivity) of all the canonical surfaces of a given manifold $M$. This number is clearly a topological invariant. A simpler question is to ask: under what conditions can the genus of a canonical surface of a manifold be lowered or raised? Lemmas 1 and 2 below do not state the most general circumstances but they are sufficient for our needs.

6. In this paragraph we shall use the following notation: Let $R$ be a canonical region, $L$ its boundary; $E_{3}, E_{3}^{\prime}$ and $\varepsilon_{3} 3$-cells with boundary spheres $S_{2}, S_{2}^{\prime}$ and $\delta_{2}$. Let $S_{2}$ be separated by a circuit into the two 2-cells $\varepsilon_{2}$ and $\varepsilon_{2}^{\prime}$, and let $E_{3}\left[E_{3}^{\prime}\right]$ be separated by two non-intersecting circuits into two 2-cells $E_{2}{ }^{1}, E_{2}{ }^{2}\left[E_{2}^{\prime 1}, E_{2}^{\prime 2}\right]$ and a band $B\left[B^{\prime}\right]$. Finally, let $B\left[B^{\prime}\right]$ be separated into two 2-cells $E_{2}^{* 1}, E_{2}^{* 2}\left[E_{2}^{\prime *_{1}}, E_{2}^{\prime *_{2}}\right]$ by two arcs, each running from one of its bounding circuits to the other.

Lemut 1a. If $\bar{\varepsilon}_{3}$ and $R+L$ haie in common only' $\bar{\varepsilon}_{2}$ on their boundaries, then $R+\varepsilon_{3}+\varepsilon_{2}$ is a canonical region w'hose boundary is $L+\varepsilon_{2}^{\prime}-\varepsilon_{2}$. 
Leмма 2a. If $\varepsilon_{3}$ is a subcomplex of $R$ and if $\mathcal{S}_{2}$ and $L$ have $\bar{\varepsilon}_{2}^{\prime}$ in common, then $R-\varepsilon_{3}-\varepsilon_{2}$ is a canonical region whose boundary is $L+\varepsilon_{2}-\varepsilon_{2}^{\prime}$.

These lemmas need no proofs. Obviously, the genus of $L$ is neither raised nor lowered by the operations of the lemmas.

We can, however, change the genus of $L$ by removing or adding a handle, $E_{3}$.

Leмma 1b. If $E_{3}$ is a subcomplex of $R$ and if $S_{2}$ and $L$ have in common $\bar{B}$, then $R-\left(E_{3}+E_{2}{ }^{1}+E_{2}{ }^{2}\right)$ is a canonical region whose boundary is $L+\left(E_{2}^{1}+E_{2}^{2}\right.$ $-B)$.

LeMma 2b. If $\bar{E}_{3}$ and $R+L$ have in common only $\bar{E}_{2}^{1}$ and $\bar{E}_{2}^{2}$ on their boundaries, then $R+\left(E_{3}+E_{2}^{1}+E_{2}^{2}\right)$ is a canonical region whose boundary is $L+\left(B-E_{2}^{1}-E_{2}^{2}\right)$.

These lemmas, too, need no proof. In the first case, the genus of $L$ is lowered by the removal of a handle, in the second, raised by the addition of a handle.

We can lower the genus of $L$ by attaching to it a 3-cell $E_{3}^{\prime}$ along a band and we can raise the genus by removing such a 3-cell, i.e. by "boring" a hole through the region. However, we can add or remove a 3-cell only under certain conditions which are stated in the lemmas below.

LEMMA 1c. Let $E_{3}^{\prime}$ and $R+L$ have no points in common and $S_{2}^{\prime}$ and $L$ have $\bar{B}^{\prime}$ in common, and let $E_{3}$ be as in Lemma $1 \mathrm{~b}$; then, if $S_{2}$ and $S_{2}^{\prime}$ have only $\bar{E}_{2}^{*_{1}} \equiv \bar{E}_{2}^{\prime *_{1}}$ in common, $R+E_{3}^{\prime}+B^{\prime}$ is a canonical region whose boundary is $L+\left(E_{2}^{\prime 1}+E_{2}^{\prime 2}-B^{\prime}\right)$.

For since, by Lemma $1 \mathrm{~b}, R-\left(E_{3}+E_{2}{ }^{1}+E_{2}{ }^{2}\right)$ is a canonical region whose boundary has the closure of the 2-cell $E_{2}^{1}+E_{2}^{2}+E_{2}^{\prime * 2}$ in common with the boundary of the 3-cell $E_{3}+E_{3}^{\prime}+E_{2}{ }^{*}$, it follows from Lemma 1a that

$$
\begin{aligned}
{\left[R-\left(E_{3}+E_{2}{ }^{1}+E_{2}{ }^{2}\right)\right]+\left[E_{3}+E_{3}^{\prime}\right.} & \left.+E_{2}{ }^{* 1}\right] \\
& +\left[E_{2}{ }^{1}+E_{2}{ }^{2}+E_{2}^{\prime * 2}\right] \equiv R+E_{3}^{\prime}+B^{\prime}
\end{aligned}
$$

is a canonical region. It is clear that the boundary of $R+E_{3}^{\prime}+B^{\prime}$ is $L+E_{2}^{\prime 1}$ $+E_{2}^{\prime 2}-B^{\prime}$.

Lemma 2c. If $E_{3}^{\prime}$ is a subcomplex of $R$ such that $S_{2}^{\prime}$ and $L$ have $\bar{E}_{2}^{\prime 1}$ and $\bar{E}_{2}^{\prime 2}$ in common, and if there exists a 3-cell $E_{3}$, subcomplex of $R$, such that $E_{2}^{*_{1}} \equiv E_{2}^{\prime *_{1}}$ and $E_{2}^{*_{2}}$ is a 2-cell on $L$, then $R-E_{3}^{\prime}-B^{\prime}$ is a canonical region whose boundary is $L-E_{2}^{\prime 1}-E_{2}^{\prime 2}+B^{\prime}$.

For, since $E_{3}+E_{3}^{\prime}+E_{2}^{\prime *_{1}}$ is a 3 -cell, it follows from Lemma 2 a that $R-\left(E_{3}+E_{3}^{\prime}+E_{2}^{\prime *_{1}}\right)-\left(E_{2}{ }^{1}+E_{2}{ }^{2}+E_{2}^{\prime *_{2}}\right)$ is a canonical region. Hence, by 
Lemma $2 \mathrm{~b}, R-\left(E_{3}+E_{3}^{\prime}+E_{2}^{\prime * 1}\right)-\left(E_{2}^{1}+E_{2}^{2}+E_{2}^{\prime * 2}\right)+E_{3}+E_{2}^{1}+E_{2}^{2} \equiv R-E_{3}^{\prime}$ $-B^{\prime}$ is a canonical region, whose boundary, as can be readily seen, is $L-E_{2}^{\prime 1}$ $-E_{2}^{\prime 2}+B^{\prime}$.

7. Let the canonical surface $L$ of a manifold $M$ divide it into the two regions $R_{1}$ and $R_{2}$; let $E$ and $F$ be canonical 2-cells of $R_{1}$ and $R_{2}$, respectively, $e$ and $f$, their boundaries. Let $E_{3}$ be a 3-cell as in Lemma $1 \mathrm{~b}$, where we put $E \equiv E_{2}^{1}$ and $R=R_{1}$. Let $A$ be a 1-cell interior to $R_{1}$ with end points on $L$. Let $E_{3}^{\prime}$ be a 3-cell as in Lemma $2 \mathrm{c}$ which is a neighborhood of $A$ in $R_{1}$. The 2-cells $E_{2}^{\prime 1}$ and $E_{2}^{\prime 2}$ will be neighborhoods on $L$ of the end points of $A$. We now have

LEMMA 1. If the canonical curves $e$ and $f$ meet once and only once, then $L+\left(E_{2}{ }^{1}+E_{2}{ }^{2}-B\right)$ is a canonical surface dividing $M$ into the two canonical regions $R_{1}-\left(E_{3}+E_{2}^{1}+E_{2}^{2}\right)$ and $R_{2}+\left(E_{3}+B\right)$.

Lemma 2. If there exists a 1-cell $A^{\prime}$ on $L$ such that $A+A^{\prime}$ bounds a 2-cell of $R_{1}$, then $L-\left(E_{2}^{\prime 1}+E_{2}^{\prime 2}-B^{\prime}\right)$ is a canonical surface dividing $M$ into the canonical regions $R_{1}-\left(E_{3}^{\prime}+B^{\prime}\right)$ and $R_{2}+\left(E_{3}^{\prime}+E_{2}^{\prime 1}+E_{2}^{\prime 2}\right)$.

The proofs of the two lemmas follow at once from Lemmas $1 \mathrm{abc}, 2 \mathrm{abc}$. We note that the effect of the first lemma is to remove a handle from $R_{1}$ (as in Lemma 1b) and to add a 3-cell to $R_{2}$ (as in Lemma 1c) and the effect of the second lemma is to remove a 3-cell from $R_{1}$ (as in Lemma 2c) and to add a handle to $R_{2}$ (as in Lemma $2 \mathrm{~b}$ ). The genus of the canonical surface is lowered in the first lemma, raised in the second.

8. Construction of the Heegaard diagram. The utility of the canonical surface and curves lies in the fact that they give us an adequate representation of the manifold. Indeed, let $L$ be a canonical surface of a manifold $M$, and let $e^{1}, \cdots, e^{p}, f^{1}, \cdots, f^{p}$ be two sets of canonical curves, boundaries of canonical sets of 2-cells of $R_{1}$ and $R_{2}$, respectively. Then, having $L, e^{1}, \cdots$, $e^{p}, f^{1}, \cdots, f^{p}$, we can dispense with the rest of $M$ entirely, for any information that can be derived from $M$ can be derived from them. To reconstruct a 3dimensional manifold, we attach 2-cells to each of the canonical curves, and then add two 3-cells in the obvious way. We thus obtain a manifold $N$ which in general will not be identical to $M$, but which must always be homeomorphic to it because of the construction.

Of great use in the study of manifolds is the fact that a model of the canonical surface and curves of any manifold can be constructed in ordinary spherical 3-space, $S_{3}$. If the canonical surface were orientable, we could immerse it in $S_{3}$ immediately; this is impossible if it is non-orientable. To treat both cases at the same time, we adopt one of the normal forms for a 2dimensional manifold which can be immersed in $S_{3}$, i.e., the plane (plus a 
point at infinity) from which the interiors of $2 p$ circles have been removed.* We call the surface $\Lambda$, its $2 p$-bounding circles, $\epsilon_{1}{ }^{i}, \epsilon_{2}^{i}, i=1, \cdots, p$. For the sake of definiteness later on, let us assume that the circles are of equal radii with centers equally spaced along a straight line.

We now establish a continuous correspondence between the points of $L$ and $\Lambda$ which is $(1,1)$ everywhere except that a point on $e^{i}$ has for image a point on $\epsilon_{1}{ }^{i}$ and a point on $\epsilon_{2}{ }^{i}$. Thus, the image of an $e$ curve will be a pair of the $\epsilon$ circles on $\Lambda$. If a particular $f^{i}$ does not meet any of the $e$ curves, then its image on $\Lambda$ will be a circuit; if it does meet the $e$ 's, then its image on $\Lambda$ will consist of a set of arcs, each joining two points on the circles. We call the circuit or aggregate of arcs corresponding to $f^{i}, \phi^{i}$.

From what has been said above, it is clear that $\Lambda, \epsilon_{1}^{i}, \epsilon_{2}^{i}$, and $\phi^{i}(i=1$, $\cdots, p)$ also serve as an adequate representation of the manifold $M$. We call this representation a Heegaard diagram of $M$.

For some purposes it is convenient to have a Heegaard diagram in which the $f$ 's are mapped on the pairs of circles, and the $e$ 's become aggregates of arcs. In such cases, we shall use another plane $\Lambda^{\prime}$, and introduce notation as needed.

To reconstruct a manifold $N$ from a Heegaard diagram $\Delta$, we first subdivide $\Lambda$, if necessary, and then construct a closed 2-dimensional manifold $L$ equivalent to $\Lambda$ where, however, a single circuit $e^{i}$ corresponds to the pair of circles $\epsilon_{1}^{i}$ and $\epsilon_{2}^{i}$. We must take care that $\epsilon_{1}^{i}$ and $\epsilon_{2}^{i}$ are matched upon $e^{i}$ with the proper orientations. We then proceed as before, successively adding the 2-cells and finally the two 3-cells.

If a Heegaard diagram is constructed from a manifold, we shall say that the manifold gives rise to the diagram and that the diagram arises from the manifold; similarly we shall say that a diagram gives rise to a manifold and that the manifold arises from the diagram when the manifold is constructed from the diagram.

9. Since a Heegaard diagram is an adequate representation of its manifold, all invariants of the latter should be obtainable from the former. We show how to get the Poincare group, homology characters, and some intersection invariants in the next section.

Since any one manifold can give rise to a great variety of diagrams, we do not seem to be any nearer the solution of the problem of the classification of manifolds. We reserve all such questions for Parts III, IV, and V; at the present we merely note that the form of a Heegaard diagram arising from a manifold is by no means unique.

* See Alexander, J. W., Normal forms for one- and tw'o-sided surfaces, Annals of Mathematics, (2), vol. 16, No. 4, June, 1915. 


\section{THE KNOWN INVARIANTS}

1. The Poincaré group. Let $\Delta$ be a Heegaard diagram of a manifold $M$, where $\Delta$ consists of $\Lambda, \epsilon_{1}^{i}, \epsilon_{2}{ }^{i}$ and $\phi^{i}, i=1,2, \cdots, p$, all defined as in Part I.

It is well known that there exist, on $\Lambda, 2 p$ circuits, $a_{i}$ and $b_{i}, i=1, \cdots, p$, all passing through a fixed point $O$ but having no other points in common and such that every. other closed curve of $\Lambda$ is deformable into a sum of the $a$ 's and $b$ 's. The $a$ 's and $b$ 's can then be taken as the generators of the Poincare group of $\Lambda$. Moreover, we can always choose the curves in such a manner that $a_{k}$ is isotopic to $\epsilon_{1}{ }^{k}$ on $\Lambda$ and $b_{k}$ consists of two arcs joining $O$ to congruent points of $\epsilon_{1}^{k}$ and $\epsilon_{2}^{k}$. We can choose a similar base on $\Lambda^{\prime}$ (another representation of $\Delta$, in which a pair of circles $\phi_{1}^{i}$ and $\phi_{2}{ }^{i}$ represents a canonical curve $f^{i}$ ), namely $c_{i}$ and $d_{i}, i=1, \cdots, p$, where $c_{k}$ is isotopic to $\phi_{1}{ }^{k}$ on $\Lambda^{\prime}$ and $d_{k}$ consists of two arcs joining $O^{\prime}$ (image of $O$ ) to congruent points of $\phi_{1}{ }^{k}$ and $\phi_{2}{ }^{k}$.

Since the surfaces $\Lambda$ and $\Lambda^{\prime}$ are representations of the same surface $L$ of $M$, we can express every curve of one base as a product of the generators of the other base, i.e., $c_{k}=a_{i_{1}}^{e_{1}} b_{i_{2}}^{\epsilon_{2}} a_{i_{3}}^{\epsilon_{i}} b_{i_{1}}^{e_{4}} \cdots$, where $i_{j}$ is one of the integers $1,2, \cdots$, $p$ and $\epsilon_{j}$ is 0,1 or -1 . Symbolically we can write

$$
\begin{aligned}
& c_{i}=\Pi_{i}{ }^{1} a b, \quad d_{i}=\Pi_{i}{ }^{2} a b, \\
& a_{i}=\Pi_{i}{ }^{3} c d, \quad b_{i}=\Pi_{i}{ }^{4} c d .
\end{aligned}
$$

The only identity relation among the generators is

$$
a_{1} b_{1} a_{1}^{-1} b_{1}^{-1} a_{2} b_{2} a_{2}^{-1} b_{2}^{-1} \cdots a_{p} b_{p} a_{p}^{-1} b_{p}^{-1}=1
$$

in case $A$ is orientable, or

$$
a_{1} b_{1} a_{1} b_{1}^{-1} a_{\varepsilon} b_{2} a_{2} b_{2} b_{2}^{-1} \cdots c_{p} b_{p} a_{p} b_{p}^{-1}=1
$$

in case $A$ is non-orientable.* There are, of course, similar expressions in terms of the c's and $d$ 's.

Let us now adjoin to $\Lambda$ the pairs of congruent 2-cells $E_{1}^{i}$ and $E_{2}^{i}$, interiors of the circles $\epsilon_{1}{ }^{i}$ and $\epsilon_{2}{ }^{i}$, where $E_{1}{ }^{i}$ and $E_{2}{ }^{i}$ correspond to the same canonical 2-cell $E^{i}$ of $R_{1}$. To note what modification the group of $\Lambda$ undergoes, we shall make use of a theorem concerning the Poincare grqup of an arbitrary 2dimensional complex $K_{2}$ : If the Poincare group of $K_{2}$ is generated by the elements

with the identity relations

$$
g_{1}, g_{2}, \cdots, g_{p}
$$

$$
r_{i}=g_{i j_{1}}^{\epsilon_{1}} g_{i j_{2}}^{\epsilon_{2}} g_{i j_{3}}^{\epsilon_{3}} \ldots=1 \quad(i=1,2, \cdots, q)
$$

* The identity relation in the non-orientable case is not one of the usual forms; it is, however, equivalent to them in the sense that we can so choose the generators of the Poincaré group that $\left(2^{\prime}\right)$ holds. 
where $g_{i j_{k}}^{\epsilon_{k}}$ is either a generating element or its inverse and we adjoin to $K_{2}$ a 2-cell $E$ having no points in common with $K_{2}$ but whose boundary is $h$, where $h$ is a circuit of $K_{2}$, then the group of $K_{2}+E$ is the same as the group of $K_{2}$ plus the additional relation

$$
r=g_{i_{1}}^{\epsilon_{1}} g_{i_{2}}^{e_{2}} g_{i_{3}}^{\epsilon_{2}} \ldots=1,
$$

where $r$ represents a curve isotopic to $h$ on $K_{2}{ }^{*}$ In particular, if $h$ is isotopic to a generator, say $g_{1}$, of the group of $K_{2}$, the group of $K_{2}+E$ can be obtained by replacing $g_{1}$ by 1 wherever it occurs in the identity relations of the group of $K_{2}$.

It follows at once that the group of $\Lambda+\sum_{i=1}^{p}\left(E_{1}^{i}+E_{2}^{i}\right)$ is generated by the $p$ elements

$$
b_{1}, b_{2}, \cdots, b_{p}
$$

with no generating relations, since $(2)$ or $\left(2^{\prime}\right)$ reduces to the unit element identically. The products (1) and $\left(1^{\prime}\right)$ take on new forms which we write symbolically as

$$
\begin{aligned}
c_{i} & =\Pi_{i}^{\prime 1} b, & d_{i} & =\Pi_{i}^{\prime 2} b \\
1 & =\Pi_{i}^{\prime 3} c d, & b_{i} & =\Pi_{i}^{\prime}{ }^{4} c d .
\end{aligned}
$$

We now add the $p$ 2-cells $F^{i}$ to $\Lambda+\sum\left(E_{1}^{i}+E_{2}^{i}\right) \equiv \Lambda+\sum E^{i}$ obtaining the 2-dimensional complex $L+\sum E^{i}+\sum F^{i}$. The products (4) and (4') take on the new forms

$$
\begin{aligned}
& 1=\Pi_{i}^{\prime \prime 1} b, \quad d_{i}=\Pi_{i}^{\prime \prime 2} b \\
& 1=\Pi_{i}^{\prime \prime 3} d, \quad b_{i}=\Pi_{i}^{\prime \prime}{ }^{4} d .
\end{aligned}
$$

From what has just been said, it is clear that the group of $\Lambda+\sum E^{i}+\sum F^{i}$ is generated by the $p$ elements (3) connected by the $p$ relations $\Pi_{i}^{\prime \prime 1} b=1$ of (5). We obtain an equivalent Poincaré group if we take for basis the $p$ elements

$$
c_{1}, c_{2}, \cdots, c_{p}
$$

connected by the $p$ relations $\Pi_{i}^{\prime \prime 3} d=1$ of $\left(5^{\prime}\right)$.

The addition of the remainder of the manifold $M$ (that is, the two 3-cells) can have no effect on the Poincaré group since a curve homotopic to a point

* If $r^{\prime}$ is another product of the $g$ 's also representing a curve isotopic to $h$ on $K_{2}$, then the group obtained by adding $r^{\prime}$ is equivalent to the group obtained by adding $r$, since $1=r^{\prime}=s r s^{-1}$, where $s$ is a product of $g^{\prime}$. 
over $M$ is first deformable over $M$ on to $L+\sum E^{i}+\sum F^{i}$ and is then homotopic to a point over $L+\sum E^{i}+\sum F^{i}$.

Hence the Poincare group $G$ of $M$ is given by

$$
\begin{aligned}
& b_{1}, b_{2}, \cdots, b_{p} \\
& \text { generators } \\
& b_{1}^{\alpha_{11}^{1}} b_{2}^{\alpha_{12}^{2}} \cdots b_{p}^{\alpha_{1 p}^{1}} b_{1}^{\alpha_{11}^{1}} \cdots b_{p}^{\alpha_{2 p}^{1}} \cdots b_{p}^{\alpha_{k, p}^{1}}=1 \\
& b_{1}^{\alpha_{11}^{2}} b_{2}^{\alpha_{12}^{3}} \cdots b_{p}^{\alpha_{1 p}^{2}} b_{1}^{\alpha_{12}^{2}} \cdots b_{p}^{\alpha_{2 p}^{2}} \cdots b_{p}^{\alpha_{k, p}^{2}}=1 \text { identity relations } \\
& b_{1}^{\alpha_{1}^{p}} b_{2}^{\alpha_{12}^{p}} \cdots b_{p}^{\alpha_{1}^{p}} b_{1}^{\alpha_{11}^{p}} \cdots b_{p}^{\alpha_{p p}^{p}} \cdots b_{p}^{\alpha_{p}^{p} p}=1
\end{aligned}
$$

where the identity relations are the products $\Pi_{i}^{\prime \prime 1}$ of (5) written out in full. There is an equivalent group in terms of the $d$ 's, but we shall not write it out.

2. The homology characters. Associated with $G^{M}$ is the group $G^{M_{c}}$ which is obtained by making all the elements commutative. $G^{M_{c}}$ is given by

$$
\begin{aligned}
& b_{1}, b_{2}, \cdots, b_{p} \quad \text { generators }
\end{aligned}
$$

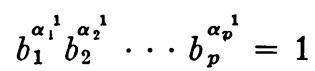

$$
\begin{aligned}
& b_{1}^{\alpha_{1}{ }^{2}} b_{2}^{\alpha_{2}{ }^{2}} \cdots b_{p}^{\alpha_{p}{ }^{2}}=1 \text { identity relations }
\end{aligned}
$$

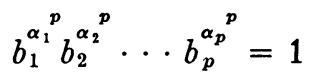

where $\alpha_{i}^{i}=\sum_{k=1}^{k_{i}} \alpha_{k_{j}}^{i}$.

Every invariant of $G^{M_{c}}$ is an invariant of $M$, the manifold giving rise to the Heegaard diagram $\Delta$. In particular, $G^{M_{c}}$ yields the 1-dimensional Betti and torsion numbers.

If $M$ is orientable, its Betti numbers are $1, p-\rho, p-\rho, 1$; if $M$ is nonorientable, its Betti numbers are $1, p-\rho, p-\rho-1,0$; where $\rho$ is the rank of the matrix $\left\|\alpha_{i}{ }^{i}\right\|$. If $M$ is orientable, it has a set of 1 -dimensional coefficients of torsion given by the invariant factors of $\left\|\alpha_{i}^{i}\right\|$; if $M$ is non-orientable, it has in addition to these a 2-dimensional coefficient of torsion equal to two.

3. The intersection numbers. We now pass to the intersection invariants of the manifold $M$. There are two types of such invariants, the Kronecker indices of the 1- and 2-cycles and the intersection 1-cycles of 2-cycles with one another. To find these invariants we need to construct a homology base for the 1- and 2-cycles.

Let us consider the boundary relations

$$
F^{i} \rightarrow \phi^{i}
$$


and the homologies

$$
\phi^{i} \sim \sum_{j} \alpha_{j}{ }^{i} b^{j}
$$

where the matrix $\left\|\alpha_{i}^{i}\right\|$ is the same as the matrix of exponents in the identity relations of $G \boldsymbol{M}_{c}$. It is well known that there exist unimodular transformations $F^{i}=\sum_{j} \mu_{j}^{i} F^{\prime}{ }^{\prime}, \phi^{i}=\sum_{i} \mu_{j}^{i} \phi^{\prime i}$ and $b^{i}=\sum_{i} \nu_{j}^{i} b^{\prime}{ }^{i}$ such that the bounding relations (6) and the homologies (7) become

$$
F^{\prime i} \rightarrow \phi^{\prime i}
$$

and

$$
\phi^{\prime i} \sim \sum_{j} \alpha_{i}^{\prime i} b^{\prime} j
$$

respectively, where $\left\|\alpha_{j}^{\prime}\right\| \|$ is a diagonal matrix. The first $\rho-\tau$ terms of the main diagonal of $\left\|\alpha_{i}^{\prime}\right\|$ are equal to 1 , the next $\tau$ terms are the invariant factors (1-dimensional coefficients of torsion) and all the other terms are zero.

Since every 1-cycle of $M$ is homologous to a linear combination of the $b$ 's, and hence to the $b^{\prime \prime}$ s, and since the 1-dimensional Betti number of $M$ is $p-\rho$, it follows that the curves

$$
b^{\prime i} \quad(i=\rho+1, \rho+2, \cdots, p)
$$

form a basis for the non-bounding 1-cycles of $M$ in this sense: if $b$ is any 1-cycle of $M$, then

$$
b \sim \sum_{i=\rho+1}^{p} a_{i} b^{\prime i}+\sum_{i=\rho-\tau+1}^{\rho} a_{i} b^{\prime i}
$$

But since some multiple of $b^{\prime i}(\rho-\tau+1 \leqq i \leqq \rho)$ bounds, it will meet any 2-cycle of $M$ zero times algebraically. Hence as far as the intersection of $b$ with a 2-cycle is concerned, only the terms of the first sum in $b$ need to be considered.

It can be shown that the 2-dimensional complexes

$$
F^{\prime i} \quad(i=\rho+1, \rho+2, \cdots, p)
$$

can serve as a base for the non-bounding 2-cycles as far as intersections are concerned. To be sure, none of the $F$ 's is a cycle, nor is any combination of them a cycle since every $\operatorname{sum} \sum \lambda_{i} F^{\prime i}$ has for boundary $\sum \lambda_{i} \phi^{\prime i}$, but it can be shown that if the complexes $F_{0}^{i}, i=\rho+1, \cdots, p$, do form a base for the non-bounding 2-cycles of $M$ in the same sense as the $b^{i}$ 's just above, then the intersection of $b^{i}$ and $F_{0}^{j}$ on $M$ is the same as the intersection of $b^{i}$ and the 
boundary of $F^{i}$ on $L$. Hence the intersection matrix of the non-bounding 1and 2-cycles of $M$ is given by

$$
\left(b^{\prime i} \cdot F^{\prime}{ }^{j}\right)
$$

$$
(i, j=\rho+1, \cdots, p)
$$

where the element in the $i$ th row and $j$ th column is the Kronecker index of $b^{\prime i}$ and $\phi^{\prime i}$ on $\Lambda$. In case $M$ is orientable, the matrix (12) is unimodular and can be transformed into a diagonal matrix by further unimodular transformations of the $b^{\prime \prime}$ s and $F^{\prime \prime}$ s.

The 2-complexes (11) also yield the intersections of the non-bounding 2-cycles among themselves. Indeed, we can compute from them a cubic matrix

$$
\left\|\sigma_{i j k}\right\| \quad(i, j=\rho+1, \rho+2, \cdots, p ; k=1,2, \cdots, p),
$$

where we can show that $\sum_{k} \sigma_{i j k} b^{k}$ is a cycle homologous to the intersection cycle of two non-bounding 2-cycles of $M$, as defined, for example, by Lefschetz in his Colloquium Lectures, Chapter IV. The $\sigma$ 's are precisely the same as those defined by Alexander in Proceedings of the National Academy of Sciences, 1924, p. 99.

They are obtained in this fashion. If $i=j$, then $\sigma_{i j k}=0$ for all $k$ 's. If $i \neq j$, we proceed as follows.

We consider a particular pair of (11), say $F^{\prime 1}$ and $F^{\prime 2}$, and a particular canonical circle, say $\epsilon_{1}{ }^{3}$. Since $F^{\prime 1}$ can be considered a two-cycle, its boundary on $\Lambda$, namely $\phi^{\prime 1}$, meets $\epsilon_{1}{ }^{3}$ zero times algebraically, if we count each intersection with its proper orientation and multiplicity. Now imagine that the curve $\epsilon_{1}{ }^{3}$ is shrunk to its center $A$. The point $A$ is met zero times algebraically by the segments of $\phi^{\prime 1}$ abutting on it.

Similarly, the point $A$ will be met zero times, algebraically, by the segments of $\phi^{\prime 2}$ incident with it.

If no segment is common to $\phi^{\prime 1}$ and $\phi^{\prime 2}$, we define the number $\sigma_{123}$ as the Kronecker index of $\phi^{\prime 1}$ and $\phi^{\prime 2}$ at the point $A$, where we take into account the multiplicity of the various branches.

Suppose now that the segment $a$ belongs to $\phi^{\prime 1}$ with the multiplicity $m_{1}$ and to $\phi^{\prime 2}$ with the multiplicity $m_{2}$. Replace $a$ by two distinct segments $a^{1}$ and $a^{2}$ lying very close to it, and let $a^{1}$ belong to $\phi^{\prime 1}$ with the multiplicity $m_{1}$ and $a^{2}$ to $\phi^{\prime 2}$ with the multiplicity $m_{2}$. If we do this for all segments common to $\phi^{\prime 1}$ and $\phi^{\prime 2}$ we are led to the former case and can define $\sigma_{123}$ as there. However, it is apparent that $\sigma_{123}$ as so defined is not unique, for the Kronecker index will depend on the method of replacing $a$ by $a^{1}$ and $a^{2}$, that is, on whether we take $a^{1}$ to the right or left of $a^{2}$. We therefore define as the $\sigma_{123}$ 
the number of smallest numerical value so obtained. As a matter of fact, the difference between any two values represents a cycle which is homologous to zero; we choose the smallest numerical value purely for convenience.

We can now define the $\sigma$ 's for all $i, j$, and $k$ in the same way. It is clear that we need not consider the canonical circles $\epsilon_{2}{ }^{i}$, since the intersections are the same as on $\epsilon_{1}^{i}$.

\section{Definitions of the moves ANd Equivalent HeggaARd diagrams}

1. Two Heegaard diagrams $\Delta$ and $\Delta^{\prime}$ with canonical curves $\epsilon^{1}, \cdots$, $\epsilon^{p}, \phi^{1}, \cdots, \phi^{p}$ and $\epsilon^{\prime 1}, \cdots, \epsilon^{\prime q}, \phi^{\prime 1}, \cdots, \phi^{\prime q}$, respectively, are said to be identical if

(a) $p=q$, and

(b) $\Delta$ and $\Delta^{\prime}$ are mapped on the same euclidean plane $\Lambda$ in such a way that each pair of circles $\epsilon_{1}^{\prime k}$ and $\epsilon_{2}^{\prime k}$ coincides in position and orientation with a pair $\epsilon_{1}{ }^{j}$ and $\epsilon_{2}{ }^{j}$ and each $\phi^{\prime k}$ is isotopic to a $\phi^{j}$ on $\Lambda$.

2. We now define a set of transformations, called moves, which operate on and modify the curves and surface of a Heegaard diagram. The moves will fall into three classes or types; the first will modify the canonical curves, the second will modify the plane $\Lambda$ by rotating a portion of it through a multiple of $\pi$, the third will modify the canonical curves and the plane $\Lambda$ by the addition (or subtraction) of canonical curves.

Type I. A. A move of Type IA simply changes the orientation of a given canonical curve.

Let $g$ be a 1-sphere on $\Lambda$ not meeting any of the circles (images of the canonical $e$ curves) and containing in its interior at least one circle, say $\epsilon_{1}{ }^{k}$, and at most two such circles. In the latter case, the two circles must be adjacent but not the two circles of a pair, i.e., images of the same $e$. Sever $\Lambda$ along $g$ and call the inner and outer lips of the cut $g$ and $g^{\prime}$, respectively. Remove the interior of $g$ from $\Lambda$ and attach it once more, this time, however, by matching corresponding points of $\epsilon_{1}^{k}$ and $\epsilon_{2}^{k}$. We have lost the canonical pair $\epsilon_{1}^{k}$ and $\epsilon_{2}^{k}$ and have gained a new pair of circuits, $g$ and $g^{\prime}$. The two cases give us the moves of Types IB and IC, i.e.,

B. $g$ contains only one circle,

C. $g$ contains two circles.

A move of Type IA, B or C is its own inverse. The effect of a move of Type IB is to replace a canonical curve by one isotopic to it. The effect of a move of Type IC is to replace a canonical curve by the "sum" of it and another canonical curve.

D. A move of Type ID is the replacement of the image on $\Lambda$ of a canonical $f$ curve, say $\phi^{i}$, by the sum of the images of $f^{i}$ and some $f^{i}$, i.e., by $\phi^{i}+\phi^{i}$. 
The move is effected by deforming $\phi^{i}$ on $\Lambda$ until it has a 1 -simplex in common with $\phi^{i}$. Then by removing this 1 -simplex we obtain a circuit $\phi^{\prime i}=\phi^{i}+\phi^{i}$, which after a slight deformation will have no points in common with $\phi^{j}$. The replacement of $\phi^{i}$ by $\phi^{\prime i}$ is the move of Type ID.

When a diagram has been modified by a move of Type IB or C, its circles will no longer be in standard form, i.e., of equal radii and equally spaced along a straight.line. However, an isotopic deformation of $\Lambda$ in $S_{3}$ will bring them into standard form. We shall always suppose this done when we operate on $\Lambda$ by a move of Type IB or C.

Type II. Let $g$ be a circuit on $\Lambda$ having no points in common with the canonical set $\{e\}$ and let $g^{\prime}$ be its position after a small isotopic deformation such that $g$ and $g^{\prime}$ have no points in common, i.e., they have the appearance of two concentric circles, where $g^{\prime}$ is interior to $g$, say. Let $B$ be the bandshaped region bounded by $g$ and $g^{\prime}$. Now rotate $g^{\prime}$ and that part of $\Lambda$ interior to it through a positive angle of $\pi$ or $2 \pi$, keeping $g$ and that part of $\Lambda$ exterior to it fixed. We note particularly that if one of a pair of canonical circles lies within $g^{\prime}$ and the other without, only one of them is rotated. The result is a distortion in the band $B$; however, if $B$ is suitably subdivided into simplexes of fine enough mesh, its structure will remain unaltered.

However, we do not wish to employ this type of transformation in its most general form but only in two certain cases when the region interior to $g^{\prime}$ contains either one or two canonical curves.

A. Rotation through a positive angle $\pi$ when the 1-sphere $g^{\prime}$ contains two consecutive circles.

B. Rotation through a positive angle $2 \pi$ when the 1 -sphere $g^{\prime}$ contains only a single circle.

Inverses of moves of Types IIA and B will be rotations through negative angles.

Type III. A move of this type adds a pair of canonical circles and a new canonical curve to the plane $\Lambda$. It is effected as follows: Let $\epsilon_{1}^{p+1}$ and $\epsilon_{2}{ }^{p+1}$ be a pair of circuits on $\Lambda$ each bounding a 2-cell, and let $\phi^{p+1}$ be an arc joining the point $P_{1}$ of $\epsilon_{1}{ }^{p+1}$ and the point $P_{2}$ of $\epsilon_{2}{ }^{p+1}$. The arc $\phi^{p+1}$ must have no other points in common with $\epsilon_{1}^{p+1}$ or $\epsilon_{2}^{p+1}$ and no points in common with the two 2-cells bounded by $\epsilon_{1}^{p+1}$ and $\epsilon_{2}^{p+1}$. Also $\epsilon_{1}^{p+1}, \epsilon_{2}^{p+1}$ and $\phi^{p+1}$ must not meet any of the other canonical curves or arcs. Then if we remove the interiors of $\epsilon_{1}{ }^{p+1}$ and $\epsilon_{2}{ }^{p+1}$ from $\Lambda$ and identify the points of $\epsilon_{1}{ }^{p+1}$ and $\epsilon_{2}{ }^{p+1}$ so that $P_{1}$ and $P_{2}$ are matched, we can add the pair $\epsilon_{1}{ }^{p+1}$ and $\epsilon_{2}{ }^{p+1}$ to our canonical circles and $\phi^{p+1}$ to our canonical arcs. Its inverse, the removal of a pair of canonical curves $\epsilon^{p+1}$ and $\phi^{p+1}$ under the conditions just described, will be denoted by III'. 
The surface $\Lambda$ seems to play a preferred rôle in the description and definition of these moves. However, all our moves are also applicable to the surface $\Lambda^{\prime}$, where the $\phi$ curves are represented by pairs of circles. It is not necessary to note the effect on $\Lambda^{\prime}$ of one of the moves operating on $\Lambda$; we only note that when we modify $\Lambda$ by a move of Type IC, $\Lambda^{\prime}$ is modified by a move of Type ID, and vice versa.

3. Let now $\Delta$ be a Heegaard diagram with the two canonical sets of curves $\{\epsilon\}$ and $\{\phi\}$. We state six lemmas whose proofs follow immediately from the definitions of the moves.

LEMma 3. If $g$ is a circuit on $\Lambda$ not meeting any of the canonical circles and containing in its interior $\epsilon_{1}{ }^{k}$ and any number of other circles but not $\epsilon_{2}{ }^{k}$, then the result of severing $\Lambda$ along $g$ and reattaching the piece so removed to $\Lambda$ along $\epsilon_{1}^{k}$ and $\epsilon_{2}^{k}$ may be obtained by successively applying moves of Type IC.

LEMma 4. If the set of curves $\left\{\epsilon^{\prime}\right\}$ is derived from the canonical set $\{\epsilon\}$ by a finite number of moves of Type $\mathrm{I}$, then the set $\left\{\epsilon^{\prime}\right\}$ is canonical.

LEMma 5. If the canonical set $\left\{\epsilon^{\prime}\right\}$ is derived from the canonical set $\{\epsilon\}$ by a finite number of moves of Type $\mathrm{I}$, then $\{\epsilon\}$ can be derived from $\left\{\epsilon^{\prime}\right\}$ by the same number of moves.

Lemma 6. If the surface $\Lambda$ and the canonical sets of curves $\{\epsilon\}$ and $\{\phi\}$ of a Heegaard diagram $\Delta$ are transformed into a surface $\Lambda^{\prime}$ with the sets of curves $\left\{\epsilon^{\prime}\right\}$ and $\left\{\phi^{\prime}\right\}$ by a finite number of moves, then $\Lambda^{\prime},\left\{\epsilon^{\prime}\right\}$ and $\left\{\phi^{\prime}\right\}$ form a Heegaard diagram.

LEMMA 7. If the Heegaard diagram $\Delta^{\prime}$ is derived from the Heegaard diagram $\Delta$ by a finite number of moves, then $\Delta$ can be derived from $\Delta^{\prime}$ also by a finite number of moves.

Lemma 8. If the Heegaard diagram $\Delta$ gives rise to the manifolds $N$ and $N^{\prime}$, then $N$ and $N^{\prime}$ are homeomorphic.

CoROLlary. If the manifold $M$ gives rise to the diagram $\Delta$, which in turn gives rise to the manifold $N$, then $M$ and $N$ are homeomorphic.

The proofs of this lemma and corollary follow at once from the construction of $N$ and $N^{\prime}$ as outlined in $\S 8$, Part I.

4. We are now in a position to give a formal definition of equivalence: two Heegaard diagrams are said to be equivalent if one can be transformed into a diagram identical to the other by a finite number of moves of Types I, II and III.

5. We prove the following theorem: 
THEOREM 1. If the equivalent Heegaard diagrams $\Delta$ and $\Delta^{\prime}$ give rise to the manifolds $N$ and $N^{\prime}$, then $N$ and $N^{\prime}$ are homeomorphic.

Since $\Delta$ and $\Delta^{\prime}$ are equivalent, $\Delta$ can be obtained from $\Delta^{\prime}$ by a finite sequence of moves of Types I, II, and III which transform $\Delta^{\prime}$ successively into the diagrams $\Delta^{1}, \Delta^{2}, \cdots, \Delta^{m} \equiv \Delta$. It is therefore necessary and sufficient to prove that at each stage the transformed diagram $\Delta^{k+1}$ gives rise to a manifold $N^{k+1}$ homeomorphic to the one, $N^{k}$, arising from the preceding diagram $\Delta^{k}$.

This is obviously true when we modify $\Delta^{k}$ by a move of Type IA, IB, or a move of Type II, hence we need only prove that the theorem holds when we employ- a move of Type IC, ID, or a move of Type III.

Let, then, $\Delta^{k}$ be transformed into $\Delta^{k+1}$ by a move of Type IC and let $\Delta^{k}$ and $\Delta^{k+1}$ give rise to the manifolds $N^{k}$ and $N^{k+1}$. We can suppose that the move replaces the canonical curve $\epsilon_{k}^{1}$ of $\Delta^{k}$ by the canonical curve $\epsilon_{k+1}^{1}$ $=\epsilon_{k}^{1}+\epsilon_{k}^{2}$. Let us retain the circuit $\epsilon_{k}^{1}$ on the diagram $\Delta^{k+1}$ and slightly deform it so that it does not meet any of the canonical curves of $\Delta^{k+1}$. We can then find in the manifold $N^{k+1}$ a 2-cell $E_{k}^{1}$ lying in the region containing the canonical 2-cells $E_{k^{i}+1}$ and whose boundary is $\epsilon_{k}^{1}$. The 2-cell $E_{k}^{1}$ will divide this region, a 3-cell, into two parts. If we unite these two parts along $E_{k+1}^{1}$ and consider $E_{k}^{1}$ as part of the boundary, we have again a 3-cell. We have not changed $N^{k+1}$ at all, but obviously from this point of view it may be considered a manifold arising from $\Delta^{k}$; hence by Lemma $8, N^{k+1}$ is homeomorphic to $N^{k}$.

A similar argument holds when $\Delta^{k}$ is transformed by a move of Type ID.

Suppose now that $\Delta^{k}$ is transformed into $\Delta^{k+1}$ by a move of Type III which adds the canonical curves $\epsilon^{p+1}$ and $\phi^{p+1}$ to the canonical curves of $\Delta^{k}$. In the manifold $N^{k+1}$ the curves $\epsilon^{p+1}$ and $\phi^{p+1}$ are such that Lemma 1 is applicable where the 2-cell $E^{p+1}$ plays the rôle of $E_{2}^{1}$ of the lemma. If we make the necessary modifications, we transform the canonical surface and regions of $N^{k+1}$ into new ones. But with these latter canonical surface and regions $N^{k+1}$ may be considered as a manifold arising from $\Delta^{k}$; hence, once more $N^{k}$ and $N^{k+1}$ are homeomorphic and the theorem is proved.

6. If, then, the Heegaard diagrams $\Delta$ and $\Delta^{\prime}$ are equivalent, i.e., if one can be transformed into the other by means of the moves, then any two manifolds $N$ and $N^{\prime}$ that arise from them are homeomorphic. To give greater justification to the definition and notion of equivalence, we must prove conversely that if two manifolds $M$ and $M^{\prime}$ are homeomorphic and they give rise to the Heegaard diagrams $\Delta$ and $\Delta^{\prime}$ then the two diagrams are equivalent. In other words, we must prove that the moves are indeed sufficient to transform $\Delta$ into $\Delta^{\prime}$. 


\section{HEEgaARd DIAgRams ARISING From a MANIFOLD}

1. Variations in a Heegaard diagram may arise in

(a) the choice of the canonical surface,

(b) the choice of the canonical 2-cells of one of the canonical regions,

(c) the choice of the canonical 2-cells of the other canonical region,

(d) the method of immersion in $S_{3}$, i.e., the method of mapping $L$ on $\Lambda$. In this section we study the relationship between any two diagrams arising from a manifold and prove (Theorems 2-8) that any two such diagrams are equivalent.

We shall use consistently the following notation: $M, M^{\prime}, N$, etc., shall denote a 3-dimensional manifold; $L$, a canonical surface, $R_{1}$ and $R_{2}$ the two canonical regions. Canonical sets of 2-cells of $R_{1}$ shall be denoted by $\{E\}$, $\left\{E^{\prime}\right\}$, etc., of $R_{2}$ by $\{F\},\left\{F^{\prime}\right\}$, etc. The canonical sets of curves (boundaries of the canonical 2-cells) will be denoted by $\{e\},\{f\}$, etc. We shall denote a Heegaard diagram by $\Delta, \Delta^{\prime}$, etc., and we shall use the notation $\Delta \equiv(\Lambda, \epsilon, \phi)$ to signify that the Heegaard diagram $\Delta$ consists of the plane $\Lambda$ (image of a canonical surface $L$ ), the pairs of circles, $\epsilon_{1}{ }^{i}, \epsilon_{2}{ }^{i}$ (images of the canonical $e$ curves), and the arcs $\phi^{i}$ (images of the canonical $f$ curves).

2. In Theorem 2 below we prove that two diagrams arising from the same canonical surface and curves are equivalent, i.e. two methods of immersing $L$, $\{e\}$ and $\{f\}$ in $S_{3}$ (see (d) of $\$ 1$ ) yield equivalent diagrams.

TheOREm 2. Let $L$ be a canonical surface of a manifold $M,\{e\}$ and $\{f\}$ canonical sets of curves of the two regions; then if $L,\{e\}$ and $\{f\}$ give rise to the Heegaard diagrams $\Delta \equiv(\Lambda, \epsilon, \phi)$ and $\Delta^{\prime} \equiv\left(\Lambda^{\prime}, \epsilon^{\prime}, \phi^{\prime}\right), \Delta$ and $\Delta^{\prime}$ are equivalent.

In this theorem $\Lambda$ and $\Lambda^{\prime}, \epsilon^{i}$ and $\epsilon^{\prime i}, \phi^{i}$ and $\phi^{\prime i}$ correspond to the same $L$, $e^{i}$ and $f^{i}$, respectively, of $M$. Let us superimpose the two planes $\Lambda$ and $\Lambda^{\prime}$; we can assume, without any loss of generality, that the canonical circles coincide. We choose our notation so that $\epsilon_{1}^{k}, \epsilon_{2}^{k}$ and $\epsilon_{1}^{\prime k}, \epsilon_{2}^{\prime k}\left(\phi^{k}\right.$ and $\left.\phi^{\prime k}\right)$ are images of the same $e^{k}\left(f^{k}\right)$ of $L$.

The circles $\epsilon_{1}^{k}, \epsilon_{2}^{k}$ and $\epsilon_{1}^{\prime k}, \epsilon_{2}^{\prime k}$ will not coincide, in general, but we shall show how to modify $\Lambda^{\prime}$ by means of our moves so that the two images of each $e$ do coincide and the two images of each $f$ are isotopic.

We regard the two planes $\Lambda$ and $\Lambda^{\prime}$ as infinitely close but distinct, as, for example, two sheets of a Riemann surface. There will be no misunderstanding then when we speak of the intersection of a curve on $\Lambda$ with a curve on $\Lambda^{\prime}$, and at the same time insist that when we modify $\Lambda^{\prime}$ by a move, no change occurs on $\Lambda$.

Let us denote by $\lambda^{i}, i=1,2, \cdots, 2 p-1$, the $2 p-1$ segments of the straight line in $\Lambda$ through the centers of the canonical circles. The segment 
$\lambda^{k}$ then joins the $k$ th and the $(k+1)$ st circles. By subdivision of $\Lambda$ we can make these segments 1 -cells of $\Lambda$. Let $\lambda^{\prime i}, i=1,2, \cdots, 2 p-1$, be the images of $\lambda^{i}$ in $\Lambda^{\prime}$. Then if $\lambda^{1}$ joins $\epsilon_{1}^{5}$ to $\epsilon_{2}{ }^{2}, \lambda^{\prime 1}$ joins $\epsilon_{1}^{\prime 5}$ to $\epsilon_{2}^{\prime 2}$.

Since $\Lambda-\sum_{i=1}^{p}\left(\epsilon_{1}^{i}+\epsilon_{2}^{i}\right)-\sum_{j=1}^{2 p-1} \lambda^{i}$ is a 2 -cell, to prove $\Delta$ is equivalent to $\Delta^{\prime}$ it is sufficient to prove that we can modify $\Lambda^{\prime}$ by our moves so that $\epsilon_{1}^{k}$ and $\epsilon_{2}^{k}$ coincide with $\epsilon_{1}^{\prime k}$ and $\epsilon_{2}^{\prime k}$, respectively, and $\lambda^{k}$ is isotopic to $\lambda^{\prime k}$. But this is quite obviously possible by means of moves IIA and IB. Indeed, let $\epsilon_{1}^{1}$ be the first circle on $\Lambda$ (reading from left to right, say). The 1-cell $\lambda^{1}$ joins $\epsilon_{1}^{1}$ to $\epsilon^{a}$, say. By a finite number of moves of Type IIA (acting on $\epsilon_{1}^{\prime 1}$ of $\Lambda^{\prime}$ ) we can make $\epsilon_{1}^{\prime 1}$ coincide with $\epsilon_{1}{ }^{1}$. Then by another finite sequence of moves we can make $\epsilon^{\prime a}$ coincide with $\epsilon^{a}$. Moreover, we can choose this sequence of moves in such a fashion that $\lambda^{\prime 1}$ loses all its intersections with all $\lambda^{i}, i \neq 1$. Then by a finite number of moves of Type IIB acting on $\epsilon^{\prime a}$, we can make $\lambda^{\prime 1}$ lose all its intersections with $\lambda^{1}$. The 1-cells $\lambda^{1}$ and $\lambda^{\prime 1}$ are now isotopic. By continuing this process, we can make each $\lambda^{k}$ isotopic to its corresponding $\lambda^{\prime k}$ and each $\epsilon_{1}^{k}\left(\epsilon_{2}^{k}\right)$ coincident with $\epsilon_{1}^{\prime k}\left(\epsilon_{2}^{\prime k}\right)$. The theorem is therefore proved.

Corollary 1. Let $\Delta \equiv(\Lambda, \epsilon, \phi)$ be a given Heegaard diagram, and let $\bar{\Delta}$ $\equiv(\bar{\Delta}, \bar{\phi}, \bar{\epsilon})$ be a diagram obtained from $\Delta$ by mapping the arcs $\phi^{i}$ of $\Lambda$ as pairs of circles on $\bar{\Lambda}$ and the pairs of circles $\epsilon_{1}{ }^{i}$ and $\epsilon_{2}{ }^{i}$ of $\Lambda$ on arcs $\bar{\phi}^{i}$ of $\bar{\Lambda}$. Then if $\bar{\Delta}^{\prime}$ $\equiv\left(\bar{\Lambda}^{\prime}, \bar{\phi}^{\prime}, \bar{\epsilon}^{\prime}\right)$ is another diagram similarly obtained, $\bar{\Delta}$ and $\bar{\Delta}^{\prime}$ are equivalent. ${ }^{*}$

CoROLLARY 2. Let the diagram $\Delta$ be equivalent to the diagram $\Delta^{\prime} ;$ then if the diagrams $\bar{\Delta}$ and $\bar{\Delta}^{\prime}$ are obtained from $\Delta$ and $\Delta^{\prime}$, respectively, as above, they are equivalent.

The proofs of these corollaries follow directly from Theorem 2. We only need to point out that a move of Type IC acting on $\Delta$ is equivalent to a move of Type ID acting on $\bar{\Delta}$.

3. Theorem 3 below states that two Heegaard diagrams arising from two choices of the canonical 2-cells of $R_{1}$ (see (b) $\$ 1$ ) are equivalent.

THEOREM 3. If $\{e\}$ and $\left\{e^{\prime}\right\}$ are two sets of canonical curves for the same region $R_{1}$ of $M$, then any diagram $\Delta \equiv(\Lambda, \epsilon, \phi)$ arising from $L,\{e\}$ and $\{f\}$ is equivalent to any diagram $\Delta^{\prime} \equiv\left(\Lambda^{\prime}, \epsilon^{\prime}, \phi^{\prime}\right)$ arising from $L,\{e\}$ and $\{f\}$.

In this theorem, $\Lambda$ and $\Lambda^{\prime}, \phi^{i}$ and $\phi^{\prime i}$ correspond to the same $L$ and $f^{i}$, respectively, of $M$, but $\epsilon$ corresponds to an unprimed $e$ whereas $\epsilon^{\prime}$ corresponds to a primed $e$.

Let $\epsilon^{\prime \prime} i$ be the image of $e^{i}$ on $\Lambda^{\prime}$. We will prove the theorem by showing how to modify $\Lambda^{\prime}$ so that the $\epsilon^{\prime \prime}$ s are replaced, one by one, by the $\epsilon^{\prime \prime \prime}$. The

* From here on, $\bar{\Delta}$, etc., will indicate not the closure of $\Delta$, etc., but a new $\Delta$, etc. 
diagram $\Delta^{\prime}$ will be then transformed into the diagram $\Delta^{\prime \prime}$. By definition, $\Delta^{\prime}$ and $\Delta^{\prime \prime}$ are equivalent, by Theorem $2, \Delta$ and $\Delta^{\prime \prime}$ are equivalent, hence $\Delta$ and $\Delta^{\prime}$ are equivalent. The process by which we modify $\Lambda^{\prime}$ will be broken up into three cases.

Case 1. We suppose first that no $\epsilon^{\prime \prime}$ meets an $\epsilon_{1}^{\prime}$ or an $\epsilon_{2}^{\prime}$ and that no $\epsilon^{\prime \prime}$ is interior to another.

The $\epsilon^{\prime \prime}$ curves will then be circuits on $\Lambda^{\prime}$ not meeting any of the circles, $\epsilon_{1}^{\prime i}$ or $\epsilon_{2}^{\prime i}$. Each $\epsilon^{\prime \prime}$ must contain at least one $\epsilon^{\prime}$ circle; for any $i$, either $\epsilon_{1}^{\prime i}$ or $\epsilon_{2}^{\prime i}$ or both are contained in an $\epsilon^{\prime \prime}$; and there is at least one $\epsilon^{\prime}$ circle not contained in any $\epsilon^{\prime \prime}$.

Let $\epsilon_{1}^{\prime k}$ be a circle not contained in any $\epsilon^{\prime \prime}$. Its partner $\epsilon_{2}^{\prime k}$ must be contained in at least one $\epsilon^{\prime \prime}$. Several subcases now arise.

Suppose first only one $\epsilon^{\prime \prime}$ circle, say $\epsilon^{\prime \prime a}$, contains $\epsilon_{2}^{\prime k}$ and that $\epsilon^{\prime \prime a}$ contains no other $\epsilon^{\prime}$. Then by a move of Type IB, in which we sever $\Lambda^{\prime}$ along $\epsilon^{\prime \prime a}$ and heal it up again by matching $\epsilon_{1}^{\prime k}$ and $\epsilon_{2}^{\prime k}$, we transform one of the $\epsilon^{\prime \prime}$ s into one of the $\epsilon^{\prime \prime \prime}$ s.

Suppose secondly that only one $\epsilon^{\prime \prime}$ circle, say $\epsilon^{\prime \prime b}$, contains $\epsilon_{2}^{\prime k}$, but that $\epsilon^{\prime \prime b}$ contains other $\epsilon^{\prime}$ circles. If $\epsilon^{\prime \prime b}$ contains only one other such circle, say $\epsilon_{1}^{\prime m}$, then by a move of Type IC by which we sever $\Lambda^{\prime}$ along $\epsilon^{\prime \prime b}$ and heal it up again by matching $\epsilon_{1}^{\prime k}$ and $\epsilon_{2}^{\prime k}$, we again transform one of the $\epsilon^{\prime \prime}$ s into one of the $\epsilon^{\prime \prime \prime}$ s. The circle $\epsilon^{\prime m}$ is now not contained in any $\epsilon^{\prime \prime}$ curve.

If $\epsilon^{\prime \prime b}$ contains more than one circle besides $\epsilon_{2}^{\prime k}$, we modify $\Lambda^{\prime}$ by a move of Type IC by which we sever $\Lambda^{\prime}$ along a circuit not meeting $\epsilon^{\prime \prime b}$ and containing $\epsilon_{2}^{\prime{ }^{k}}$ and one other circle and then patch it up by matching $\epsilon_{1}^{\prime k}$ and $\epsilon_{2}^{\prime k}$. We thus obtain a new configuration in which there are one fewer circles interior to $\epsilon^{\prime \prime b}$. One of the new canonical circles is interior and the other is exterior to $\epsilon^{\prime \prime b}$. By repeating this process we can remove the circles interior to $\epsilon^{\prime \prime b}$ one by one until only two remain, and then we are back to the preceding case.

By a finite number of steps we can modify $\Lambda^{\prime}$ so that all the $\epsilon^{\prime \prime}$ 's become $\epsilon^{\prime \prime \prime}$ 's. Hence, $\Delta^{\prime}$ is transformed into a diagram $\Delta^{\prime \prime}$ representing $L,\{e\}$ and $\{f\}$, and is therefore equivalent to $\Delta$ by Theorem 2 , as we wished to prove.

Case 2. Let no $\epsilon^{\prime \prime}$ meet an $\epsilon^{\prime}$ circle, but suppose that some $\epsilon^{\prime \prime \prime}$ 's are interior to others.

Suppose first that the $\epsilon^{\prime \prime}$ 's form "nests" of circuits, i.e. if $\epsilon^{\prime \prime a}$ contains $\epsilon^{\prime \prime b}$ and $\epsilon^{\prime \prime c}$, then either $\epsilon^{\prime \prime b}$ contains $\epsilon^{\prime \prime c}$ or $\epsilon^{\prime \prime c}$ contains $\epsilon^{\prime \prime b}$. By considerations entirely analogous to those of Case 1 we can modify $\Lambda^{\prime}$ so that the innermost $\epsilon^{\prime \prime}$ of a nest is changed into an $\epsilon^{\prime}$ and hence the nest has one fewer $\epsilon^{\prime \prime}$ curves. After a finite number of steps we are led back to Case 1.

Now suppose that the $\epsilon^{\prime \prime \prime}$ s are contained in other $\epsilon^{\prime \prime}$ 's in a general fashion. 
Several cases arise, but it is not necessary to go into details. We can always transform $\Lambda^{\prime}$ so that the $\epsilon^{\prime \prime \prime}$ 's are transformed into nests of curves and we are back to the former case.

Case 3. We suppose that the $\epsilon^{\prime \prime \prime}$ s do meet the $\epsilon^{\prime \prime}$ s.

Let us examine, first of all, the nature of the intersection of an unprimed canonical 2-cell, say $E^{i}$, with a primed canonical 2-cell, say $E^{\prime k}$, in the manifold $M$. The intersection will consist of a number of 1-cells and circuits. By a slight deformation of $E^{\prime k}$ and its boundary $e^{\prime k}$ we can arrange so that $e^{\prime k}$ meets $e^{j}$, the boundary of $E^{j}$, only in a finite number of points, such that the end points of any 1-cell common to $E^{i}$ and $E^{\prime k}$ are distinct.

Keep $j$ fixed and let $k$ run from 1 to $p$. We obtain on $e^{i}$ a certain number of points, grouped into pairs, where each pair is the boundary of a 1-cell common to $E^{i}$ and some $E^{\prime}$ and no pair "separates" another on $e^{i}$. Hence there is at least one pair, say $P_{1}$ and $P_{2}$, such that one of the two arcs into which $P_{1}$ and $P_{2}$ divide $e^{j}$ contains no other intersection point. Let us call this arc $a$. The points $P_{1}$ and $P_{2}$ are on some $e^{\prime}$, say $e^{\prime k}$.

We now return to the Heegaard diagram $\Delta^{\prime}$ on $\Lambda^{\prime}$. The canonical curve $e^{\prime k}$ is represented by the pair of circles $\epsilon_{1}^{\prime k}$ and $\epsilon_{2}^{\prime k}$; each circle has on it the images of $P_{1}$ and $P_{2}$, which we continue to call $P_{1}$ and $P_{2}$, and $a$ is now mapped on a 1-cell $\alpha$ of $\Lambda^{\prime}$ joining $P_{1}$ and $P_{2}$ of $\epsilon_{2}^{\prime k}$, say. The arc $\alpha$ meets no other circle.

We now show that we can always modify $\Lambda^{\prime}$ so as to lose the two intersections $P_{1}$ and $P_{2}$. The circle $\epsilon_{2}^{\prime k}$ and the arc $\alpha$ divide $\Lambda^{\prime}$ into two regions. Let $A$ be the one which does not contain $\epsilon_{1}^{\prime k}$. Several cases arise. Suppose first that $A$ contains no canonical circle at all. Then, obviously, $\alpha$ can be deformed so that the intersections $P_{1}$ and $P_{2}$ are lost.

Suppose next that $A$ contains only one canonical circle. Let $g$ be a circuit in $\Lambda^{\prime}-A$ which lies very close to $\alpha$ and that part of $\epsilon_{2}^{\prime k}$ bounding $\Lambda^{\prime}-A$. Then if we modify $\Lambda^{\prime}$ by a move of Type IC in which we sever $\Lambda^{\prime}$ along $g$ and patch it up again by matching $\epsilon_{1}^{\prime k}$ and $\epsilon_{2}^{\prime k}$, we find that the two intersections $P_{1}$ and $P_{2}$ have disappeared.

Suppose, finally, that $A$ contains several canonical circles. Choose $g$ as before and again sever $\Lambda^{\prime}$ along it and patch it up again along corresponding points of $\epsilon_{1}{ }^{k}$ and $\epsilon_{2}{ }^{k}$. Again, we lose the two intersections, $P_{1}$ and $P_{2}$, and, by Lemma 3, this operation is the product of moves of Type IC. Hence since we can remove all the intersections two by two this case is reduced to the former and the theorem is completely proved.

4. Theorem 4 states that two Heegaard diagrams arising from two choices of the canonical 2-cells of $R_{2}$ (see (c), §1) are equivalent. In the previous theorem, the circles on $\Lambda$ and $\Lambda^{\prime}$ were images of different $e$ 's; in this theorem, the $\phi$ 's are images of different $f$ 's. 
TheOREM 4. If $\{f\}$ and $\left\{f^{\prime}\right\}$ are two sets of canonical curves for the same region $R_{2}$ of $M$, then any diagram $\Delta \equiv(\Lambda, \epsilon, \phi)$ arising from $L,\{e\}$ and $\{f\}$ is equivalent to any diagram $\Delta^{\prime} \equiv\left(\Lambda^{\prime}, \epsilon^{\prime}, \phi^{\prime}\right)$ arising from $L,\{e\}$ and $\left\{f^{\prime}\right\}$.

This theorem follows at once from Corollaries 1 and 2 of Theorem 2 and Theorem 3 by mapping $\Delta$ and $\Delta^{\prime}$ on $\bar{\Delta}$ and $\bar{\Delta}^{\prime}$ as in Corollary 1 .

5. We have proved, Theorems 2, 3, and 4, that any two Heegaard diagrams arising from a manifold are equivalent, provided that we chose the same canonical surface in each case. We have left to prove that any two diagrams whatsoever arising from a manifold are equivalent (see (a), §1). To prove this we make use of a special canonical surface whose construction is given below.

6. Construction of the special canonical surface. Let $M$ be a manifold which we assume simplicial, $G$ the linear graph consisting of all the 0 - and 1simplexes of $M, H$ a subcomplex of $G$. Let $M^{\prime}$ be the first derived complex of $M$, and $M^{\prime \prime}$ the first derived complex of $M^{\prime}$; and let $G^{\prime}, G^{\prime \prime}, H^{\prime}, H^{\prime \prime}$ be the induced subdivisions on $G$ and $H$, respectively. It may happen that the boundary $\dagger$ of the $M^{\prime \prime}$-neighborhood of $H$ is a canonical surface, in which case we call it a special canonical surface and any diagram arising from it a special Heegaard diagram. In particular, if $H$ is $G$ itself, we prove

Lемма 9. The boundary, $L$, of the $M^{\prime \prime}$-neighborhood of $G$ is a special canonical surface.

Let $M^{*}$ be the dual of $M, G^{*}$ the linear graph consisting of all the 0 - and 1-cells of $M^{*}$, and $G^{* \prime}$ the subdivision of $G^{*}$ induced by $M^{\prime}$. Further, let $R_{1}$ and $R_{2}$ be the $M^{\prime \prime}$-neighborhoods of $G$ and $G^{*}$, respectively. We prove that $L$ is a canonical surface by showing

(a) that every 3-simplex of $M^{\prime \prime}$ belongs either to $R_{1}$ or $R_{2}$;

(b) $L$ is the common boundary of $R_{1}$ and $R_{2}$, and is a closed and connected 2-dimensional manifold;

(c) $R_{1}$ and $R_{2}$ are canonical regions.

It then follows from our definitions (Part I, \$4) that $L$ is a canonical surface dividing $M$ into the canonical regions $R_{1}$ and $R_{2}$.

(a) Let $A_{0}{ }^{i}$ be the vertices of $M ; B_{1}{ }^{j}, B_{2}^{k}, B_{3}{ }^{l}$ the vertices of $M^{\prime}$ on the 1-, 2- and 3-cells of $M$, respectively, and $C_{1}{ }^{r}, C_{2}{ }^{s}, C_{3}{ }^{t}$ the vertices of $M^{\prime \prime}$ on the 1-, 2- and 3-cells of $M^{\prime}$, respectively. Every 3-simplex of $M^{\prime}$ is of the form $A_{0} B_{1} B_{2} B_{3}$, every 3 -simplex of $M^{\prime \prime}$ is of the form $A_{0} C_{1} C_{2} C_{3}$ or $B_{a} C_{1} C_{2} C_{3}$, where $a=1,2$, or 3 . Hence every 3-simplex of $M^{\prime \prime}$ is incident with an $A_{0}$ or a $B_{1}$, i.e. a vertex of $G^{\prime}$ or else with a $B_{2}$ or a $B_{3}$, i.e. a vertex of $G^{* \prime}$. That is, every 3simplex of $M^{\prime \prime}$ belongs to $R_{1}$ or to $R_{2}$.

$\dagger$ See second footnote on page 89. 
(b) In any 3-simplex, $\sigma_{3}{ }^{i}$, of $M^{\prime}$, the component 3-simplexes of $R_{1}$ and $R_{2}$ are grouped as the simplexes of $\$ 2$ of Part I, hence in any 3-simplex of $M^{\prime}$, the boundaries of $R_{1}$ and $R_{2}$ have in common a 2-cell which we call $E_{2}^{i}$. The boundary of $E_{2}^{i}$ is a circuit which may be thought of as composed of four 1-cells, each on one of the faces of $\sigma_{3}^{i}$. Let us call the 1-cell on the 2-simplex $\sigma_{2}{ }^{i}, E_{1}{ }^{i}$. It is quite clear that the incidence matrix of the 2- and 3-simplexes of $M^{\prime}$ is the same as the incidence matrix of all the 1- and 2-cells $E_{1}{ }^{i}$ and $E_{2}{ }^{i}$. It follows at once from the fact that $M$ is a manifold that $L=\sum E_{2}{ }^{i}$ (the sum being taken over all $i$ 's) is a closed, connected 2-dimensional cellular manifold, from which it can be deduced that, upon subdivision, $L$ will become a closed, connected 2-dimensional simplicial manifold. Furthermore, it follows that $L$ is orientable or non-orientable according as $M$ is orientable or non-orientable, and conversely.

(c) Since the $M^{\prime \prime}$-neighborhood of a 1-simplex of $M$ is a 3-cell, the $M^{\prime \prime}$ neighborhood of any tree of 1-simplexes of $M$ is also a 3-cell. Let the linear graph $G$ contain $p$ independent circuits; then the removal of $p$ properly chosen 1 -simplexes, say $\sigma_{1}^{1}, \sigma_{1}^{2}, \cdots, \sigma_{1}^{p}$, from $G$ will reduce it to a tree $T$. Let $B_{1}^{i}$ be the vertex of $M^{\prime}$ on $\sigma_{1}^{i}(i=1, \cdots, p)$ and let $E^{i}$ be the aggregate of 2simplexes of $M^{\prime \prime}$ that lie in the 2-cell of $M^{*}$ dual to $\sigma_{1}{ }^{i}$ and are incident with $B^{i}(i=1, \cdots, p)$. The $M^{\prime \prime}$-neighborhood of $T$ is a 3 -cell and if we add to it the remainder of the $M^{\prime \prime}$-neighborhood of $G$ excepting the 2-cells $E^{1}, \cdots$, $E^{p}$, we obtain a 3-cell. Hence $R_{1}$ is a canonical region.

By exactly the same argument it can be shown that $R_{2}$ is also a canonical region. It therefore follows that $L$ is a canonical surface dividing the manifold $M$ into the two canonical regions, $R_{1}$ and $R_{2}$, as we wished to prove.

7. We have proved incidentally that $L$ and $M$ are together orientable or non-orientable. Also, since the number of 2-cells which when removed reduce $R_{1}$ to a 3-cell is equal to the maximum number of non-intersecting circuits that can be drawn on $L$ without disconnecting it, it follows that the number of 2-cells removed from $R_{1}$ is equal to the number removed from $R_{\mathbf{2}}$. In other words, the cyclomatic number of $G$ is equal to the cyclomatic number of $G^{*} \dagger$.

8. We prove the following lemma:

Lemma 10. Let $H$ and $J$ be subcomplexes of the linear graph $G$ of a manifold $M$ such that it is possible to build up $B$ from $J$ by successively adding to $J$ closed 1-simplexes of $G$ in such fashion that at every step the 1-simplex which is being added either has one and only one end point in common with the subgraph already built up or else is the third side of a 2-simplex of which the other two sides

$\dagger$ The cyclomatic number of a linear graph is the number of independent 1-circuits on it, i.e. the minimum number of 1 -simplexes that can be removed which reduce the graph to a tree. 
already belong to the subgraph. Then if the boundary of the $M^{\prime \prime}$-neighborhood of $J$ is a canonical surface, so is the boundary of the $M^{\prime \prime}$-neighborhood of $H$, and conversely.

The proof of this lemma follows by induction. Suppose that at the $n$th step we have built up the subgraph $J_{n}$ from $J$ and that the boundary of the $M^{\prime \prime}$-neighborhood of $J_{n}$ is a canonical surface of $M$. We add the closed 1simplex $\sigma_{1}^{n+1}$ to $J_{n}$, obtaining the subgraph $J_{n+1}$. By hypothesis, either $\sigma_{1}^{n+1}$ has only one end point in common with $J_{n}$, or else it completes a triangle, of which the other two sides are already in $J_{n}$. It is obvious in the first case that the boundary of the $M^{\prime \prime}$-neighborhood of $J_{n+1}$ is a canonical surface of $M$.

Let then $\sigma_{1}^{n+1}$ complete a triangle of which the other two sides belong to $J_{n}$. Then if we call $R_{2}$ the $M^{\prime \prime}$-neighborhood of $J_{n}, L$ its boundary and $R_{1}$ the remainder of $M$, that part of $\sigma_{1}{ }^{n+1}$ lying in $R_{1}$ can play the rôle of $A$ of Lemma 2. Hence, applying Lemma 2 , we obtain a new canonical surface of $M$ which is precisely the boundary of the $M^{\prime \prime}$-neighborhood of $J_{n+1}$. It follows that the boundary of the $M^{\prime \prime}$-neighborhood of $H$ is a canonical surface of $M$.

Conversely, let us suppose that the boundary of the $M^{\prime \prime}$-neighborhood of $J_{n+1}$ is a canonical surface. Again, if the 1-simplex $\sigma_{1}{ }^{n+1}$ which was added to $J_{n}$ to form $J_{n+1}$ has only one end point in common with $J_{n}$, then the boundary of the $M^{\prime \prime}$-neighborhood of $J_{n}$ is a canonical surface of $M$.

In the second case, let us call that part of the 2-simplex whose boundary $\sigma_{1}^{n+1}$ completes lying in $R_{1}, E$, and $F$ that part of the 2-cell dual to $\sigma_{1}^{n+1}$ lying in $R_{2}$. We can choose $E$ and $F$ canonical 2-cells of $R_{1}$ and $R_{2}$, respectively, and so arrange that their boundaries (which meet once and only once) do not meet any of the other canonical curves. Lemma 1 is then applicable, and therefore the boundary of the $M^{\prime \prime}$-neighborhood of $J_{n}$ is a canonical surface of $M$. It follows that the boundary of the $M^{\prime \prime}$-neighborhood of $J$ is a canonical surface of $M$ and the lemma is proved.

9. Let $L$ and $L^{\prime}$ be the boundaries of the $M^{\prime \prime}$-neighborhoods of $H$ and $J$, respectively, as above, and suppose that one (and hence the other) is a canonical surface. As an immediate consequence of Lemma 10 we have

Corollary 1. Any special Heegaard diagram arising from $L$ is equivalent to any special Heegaard diagram arising from $L^{\prime}$.

The proof follows at once from the fact that when we pass from $J_{n}$ to $J_{n+1}$, we either do not change the Heegaard diagram at all, or else we modify it by a move of Type III.

10. At this point it becomes necessary to restrict the notion of homeomorphism. Let $M$ and $M_{0}$ be two manifolds which are not only homeomorphic 
but also equivalent in the sense of semi-linear analysis situs, i.e., it is possible to subdivide each of them so that the subdivisions have identical structures (cf. Alexander, J. W., The combinatorial theory of complexes, Annals of Mathematics, (2), vol. 31 (1930)). $M$ and $M_{0}$ will then be called equivalent manifolds.

From this definition and from Lemma 10 and its corollary follows at once

Theorem 5. If $M$ and $M_{0}$ are equivalent manifolds, $G$ and $G_{0}$ their linear graphs, $L$ and $L_{0}$ the boundaries of the $M^{\prime \prime}$ - and $M_{0}^{\prime \prime}$-neighborhoods of $G$ and $G_{0}$, respectively, then any special $H$ eegaard diagram of $M$ arising from $L$ is equivalent to any special Heegaard diagram of $M_{0}$ arising from $L_{0}$.

11. We have one more theorem to prove before we can prove our objective, Theorem 7 .

THEOREM 6. Let $G$ be the linear graph of a manifold $M, L_{0}$ the boundary of the $M^{\prime \prime}$-neighborhood of $G$, and $L$ any canonical surface whatsoever of $M$. Then any Heegaard diagram $\Delta$ of $M$ arising from $L$ is equivalent to any special Heegaard diagram $\Delta_{0}$ arising from $L_{0}$.

Let us note that if the canonical surface $L$ is isotopic to a surface $L^{\prime}$, then $L^{\prime}$ is also a canonical surface of $M$.

Let $L$ divide the manifold $M$ into the canonical regions $R_{1}$ and $R_{\mathbf{2}}$ and let $E_{2}{ }^{1}, \cdots, E_{2}{ }^{p}$ be a set of canonical 2-cells for $R_{1}$. After subdivision of $M$, if necessary, we can group the simplexes of $M$ into cells, so that $R_{1}$ is composed of $p+13$-cells, $E_{3}^{1}, \cdots, E_{3}^{p+1}$, where $E_{3}^{i}$ is bounded by the 2-cell $E_{2}^{i}$ and $E_{2}^{\prime i}$ (a 2-cell isotopic to $E_{2}^{i}$ ) and 2-simplexes on $L$, for $i=1,2, \cdots, p$; and $E_{3}{ }^{p+1}$ is a 3 -cell bounded by $\sum\left(E_{2}{ }^{i}+E_{2}^{\prime i}\right)$ and simplexes on $L$. By elementary operations we can further change the structure of $M$ so that all of the 3-cells $E_{3}{ }^{1}, \cdots, E_{3}{ }^{p+1}$ and all of the 2-cells $E_{2}{ }^{1}, \cdots, E_{2}{ }^{p}, E_{2}^{\prime}{ }^{1}, \cdots, E_{2}^{\prime p}$, become stars of simplexes having for centers the 0 -cells $E_{0}^{1}, \cdots, E_{0}{ }^{p+1}, P_{0}^{1}, \cdots$, $P_{0}{ }^{p}$, and $P_{0}^{\prime 1}, \cdots, P_{0}^{\prime p}$, respectively. The manifold $M$ is thus transformed into an equivalent manifold $N$. Let $L_{n}$ be the image of $L$ in $N$.

Consider the linear graph $H$ of $N$, where

$$
H=\sum_{i=1}^{p}\left(E_{0}^{p+1} P_{0}^{i}+P_{0}{ }^{i} E_{0}{ }^{i}+E_{0}{ }^{i} P_{0}^{\prime i}+P_{0}^{\prime i} E_{0}^{p+1}\right)
$$

in which $E_{0}^{p+1} P_{0}^{i}$, etc., stands for the 1-simplex joining $E_{0}^{p+1}$ and $P_{0}^{i}$. By construction, $G_{n}$, the linear graph of $N$ can be built up from $H$ as is required in Lemma 10. Hence any special Heegaard diagram of $N$, say $\Delta_{N}$, that arises from the boundary of the $N^{\prime \prime}$-neighborhood of $G_{n}$ is equivalent to any special Heegaard diagram that arises from the boundary of the $N^{\prime \prime}$-neighborhood of 
$H$. But this latter surface is isotopic to $L_{n}$. Hence $\Delta_{N}$ is equivalent to any Heegaard diagram arising from $L_{n}$. But since $L_{n}$ is the image of $L, \Delta$ is such a diagram, hence $\Delta$ and $\Delta_{N}$ are equivalent. But by Theorem $5, \Delta_{N}$ and $\Delta_{0}$ are equivalent, therefore $\Delta$ and $\Delta_{0}$ are equivalent as we wished to prove.

12. From Theorems $2,3,4,5$, and 6 follows the all inclusive theorem

THEOREM 7. If $\Delta$ and $\Delta^{\prime}$ are any two Heegaard diagrams whatsoever arising from a manifold $M$, then $\Delta$ and $\Delta^{\prime}$ are equivalent.

\section{THE GENERAL THEOREM}

1. Several theorems follow immediately from those of Part IV.

Theorem 8. If the equivalent manifolds $M$ and $N$ give rise to the Heegaard diagrams $\Delta$ and $\Delta^{\prime}$, then $\Delta$ and $\Delta^{\prime}$ are equivalent.

THEOREM 9. If the diagram $\Delta$ gives rise to the manifold $M$, which in turn gives rise to the diagram $\Delta^{\prime}$, then $\Delta$ and $\Delta^{\prime}$ are equivalent.

From Theorem 9 and the preceding theorems follows

THEOREM 10. If the Heegaard diagrams $\Delta$ and $\Delta^{\prime}$ give rise to equivalent manifolds, then $\Delta$ and $\Delta^{\prime}$ are equivalent.

TheOREM 11. If the manifolds $M$ and $N$ give rise to equivalent diagrams, then $M$ and $N$ are equivalent.

From Theorems 1, 8, 10, and 11 follows the general theorem

THEOREM 12. Equivalent manifolds give rise to and arise from equivalent Heegaard diagrams and equivalent Heegaard diagrams give rise to and arise from equivalent manifolds.

The problem of determining when two given 3-dimensional manifolds are equivalent (in the sense that we can pass from one to the other by elementary operations) is thus reduced to the problem of determining when two given Heegaard diagrams are equivalent (in the sense that we can pass from one to the other by means of the moves defined in Part III).

Princeton Untversity, Princeton, N. J. 